\title{
Effects of Ion Atomic Number on Single-Event Gate Rupture (SEGR) Susceptibility of Power MOSFETs
}

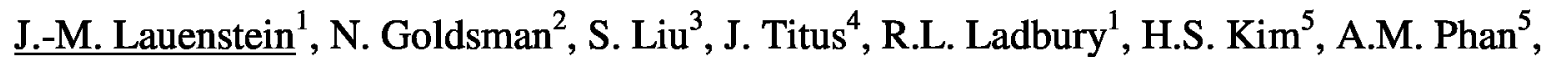 \\ M. Zafrani ${ }^{3}$, and P. Sherman ${ }^{3}$ \\ ${ }^{1}$ NASA Goddard Space Flight Center \\ ${ }^{2}$ University of Maryland, College Park \\ ${ }^{3}$ International Rectifier Corporation \\ ${ }^{4}$ Naval Surface Warfare Center \\ ${ }^{5}$ MEI Technologies
}

\section{5-WORD ABSTRACT}

The relative importance of heavy-ion interaction with the oxide, charge ionized in the epilayer, and charge ionized in the drain substrate, on the bias for SEGR failure is experimentally investigated.

\section{Corresponding (and Presenting) Author: \\ Jean-Marie Lauenstein, NASA-GSFC, Building 22, room 58, code 561.4, GREENBELT, MD 20771 \\ (USA), phone: 301-286-5592, fax: 301-286-4699, email: jean.m.lauenstein@nasa.gov \\ Contributing Authors: \\ Neil Goldsman, Department of Electrical Engineering, University of Maryland, 2453 A.V. Williams Building, COLLEGE PARK, MD 20742 (USA), phone: 301-286-3648, fax: 301-314-9281, email: neil@umd.edu}

Sandra Liu, International Rectifier Corporation, 1521 Grand Avenue, EL SEGUNDO, CA 90245 (USA) phone: 310-726-8306, fax: 310-563-1479, email: sliu1@irf.com

Jeffrey L. Titus, NAVSEA Crane Division, CRANE, IN 47522 (USA), phone: 812-854-1617, email: jeffrey.titus@navy.mil

Raymond L. Ladbury, NASA-GSFC, Building 22, room 54, code 561.4, GREENBELT, MD 20771 (USA), phone: 301-286-1030, fax: 301-286-4699, email: raymond.1.1adbury@nasa.gov

Hak S. Kim, c/o NASA-GSFC, Building 22, room 56, code 561.4, GREENBELT, MD 20771 (USA), phone: 301-286-1023, fax: 301-286-4699, email: hak.s.kim@nasa.gov

Anthony M. Phan, c/o NASA-GSFC, Building 22, room 072, code 561.4, GREENBELT, MD 20771 (USA), phone: 301-286-1239, fax: 301-286-4699, email: anthony.m.phan@nasa.gov

Max Zafrani, International Rectifier Corporation, 205 Crawford Street, Leominster, MA 01453 (USA), phone: 978-514-6187, fax: 978-537-4246, email: mzafran1@irf.com

Phillip Sherman, International Rectifier Corporation, 8845 Irvine Center Drive Suite 101, Irvine, CA 92618 (USA), phone: 949-453-1008x220, fax: 949-453-8748, email: psherma1@irf.com

\section{Session Preference: Basic Mechanisms of Radiation Effects in Electronic Materials and Devices Presentation Preference: Poster}

Abstract received Feb. 4, 2011. The authors would like to thank the NASA Electronic Parts and Packaging (NEPP) program, International Rectifier Corporation, and the Defense Threat Reduction Agency (DTRA) for support of this research. 


\section{INTRODUCTION}

Single-event gate rupture (SEGR) remains a key failure mode in vertical power MOSFETs flown in space-based missions. These devices are vulnerable to this failure mode when biased in the off state. Fig. 1 shows an illustration of a typical n-type VDMOS. The lightly-doped epitaxial layer can range from around $10 \mu \mathrm{m}$ to $120 \mu \mathrm{m}$ thick for devices with $100 \mathrm{~V}$ to $1000 \mathrm{~V}$ breakdown ratings. It is this thickness combined with the light doping that permits the formation of a large depletion region needed to hold off such high drain voltages, preventing high fields from developing in the silicon or gate oxide. Beneath this epilayer region is the highly doped drain substrate which lowers the drain contact resistance, helps to remove heat, and mechanically strengthens the die. Due to this high substrate doping concentration, the drain voltage is transferred with minimal loss from the drain contact to the base of the epilayer.

As first suggested by Fisher [1], the mechanisms of SEGR involve both the heavy-ion interaction with the gate oxide and the charge ionization in the epitaxial layer of the device. The passage of the ion through the gate oxide temporarily reduces the electric field required for dielectric breakdown; the ionized charge within the epilayer collapses the depletion region, allowing a greater portion of the high off-state drain voltage to fall across the gate oxide. More recently, it has been suggested that the charge ionized within the highly-doped drain substrate region also contributes to the maximum transient electric field across the oxide [2]. The following work seeks to enhance our understanding of the importance of these mechanisms relative to one another, as well as the importance of the ion atomic number versus ion linear energy transfer (LET).

The relative contributions of the different mechanisms to SEGR in a vertical power MOSFET are experimentally assessed through careful selection of monoenergetic ion beams based upon their average LET within the device epilayer. In this way, the threshold drain-source voltage (Vds) at which SEGR occurs can be compared for ions of differing atomic numbers $(\mathrm{Z})$ that ionize the same average total charge within the epilayer. In addition, two ion beams are chosen such that the ion with lower atomic number ionizes on average slightly more charge throughout the epilayer and a substantial portion of the substrate than does the ion with higher atomic number. Conclusions are drawn from analysis of the ion beam species and energy deposition profiles as a function of the threshold Vds determined for the given ion beam, and from expected behavior based upon the Titus-Wheatley formula [3], which is validated for the power MOSFET used in this work.

\section{EXPERIMENTAL METHODS}

A radiation-hardened $200 \mathrm{~V}$ n-type vertical power MOSFET (VDMOS) was used for these experiments. Samples came from two wafers having the same lot date code. Heavy-ion test data were taken at the Texas A\&M University Cyclotron Facility (TAMU). For each sample, the gate-source bias was held at $-10 \mathrm{~V}$ to assure that SEGR would occur during exposure to lighter, lower-LET ions. At this bias, effects of multiple proximal ion impacts are reduced [4, 5], making the data easier to interpret. Vds was incremented in 5-volt steps; at each step, the sample was irradiated until either the sample failed or a fluence of $3 \times 10^{5}$ ions $/ \mathrm{cm}^{2}$ was reached. A post-irradiation gate stress test was then performed to reveal any latent damage to the gate oxide. Failure was defined by the gate leakage current exceeding the 100 $\mathrm{nA}$ vendor specification for Igss.

The ion LET versus penetration depth in silicon is plotted in Fig. 2 for the six monoenergetic ion beams selected for this study. The ion species and energies were chosen to yield two pairs of beams having similar incident LETs and total charge ionization within the sample epilayer, and one pair in which the lower- $Z$ ion yielded a higher LET throughout the epilayer and the initial portion of the highlydoped drain substrate. Table I provides the surface-incident LET, LET at the oxide, mean LET in the epilayer region, and total charge ionized within the epilayer, as calculated with the OMERE, v. 3.4.5.0, Equivalent LET software module based on SRIM 2006 [6, 7].

\section{RESULTS}


The threshold Vds for SEGR was determined for six different monoenergetic heavy-ion beams. For four of these beams, the ions and energies were chosen to yield pairs that would on average ionize the same total charge of either $7.9 \mathrm{pC}$ or $15.5 \mathrm{pC}$ within the sensitive epilayer of the samples. In addition, one heavy-ion beam in each pairing has minimal penetration into the substrate, whereas the other beam has significant penetration into the substrate. In this way, the effect of ion LET within the epilayer was dampened to reveal any ion species or substrate effects on SEGR susceptibility. In this abstract, we describe only the results from the first pair, showing plots of results of both pairs.

The first pairing consisted of irradiations by either $422 \mathrm{MeV} \mathrm{Cu}(Z=29)$ or $1089 \mathrm{MeV} \mathrm{Kr}(Z=36)$. Three or four samples, respectively, were irradiated at a fixed $-10 \mathrm{Vgs}$, with the threshold Vds for SEGR found by incrementing Vds by $5 \mathrm{~V}$ per beam run. Due to the small sample size and the interval nature of the data from the experiments in this study, all data were analyzed as follows: We assume that for each ion species and energy, the SEGR failure Vds interval for the device tested has a normal distribution from part-to-part variability. The method of maximum likelihood was then employed to identify the mean $(\mu)$ and standard deviation $(\sigma)$ best fitting the experimental data. To further account for the limited data set and hence the unknown extent of part-to-part variability, we use the standard deviation at the boundary of the 95\% confidence level (CL) instead of this best fit value, using the Chi-square value for 2 degrees of freedom $(\mu$ and $\sigma)$.

Fig. 3 plots these means for the $\mathrm{Cu}$ and $\mathrm{Kr}$ data, with error bars indicating one standard deviation from the mean at the boundary of the $95 \% \mathrm{CL}$. As can be seen for the data taken at $-10 \mathrm{Vgs}$, despite both ions on average ionizing equal amounts of charge within the epilayer, SEGR occurs at a lower Vds under irradiation with the heavier $\mathrm{Kr}$ ion. The difference in the mean Vds for SEGR is significant at the 95\% CL. As shown in Fig. 3, we further characterized the effect of $\mathrm{Cu}$ versus $\mathrm{Kr}$ ions by irradiating two additional samples with $422 \mathrm{MeV} \mathrm{Cu}$, holding Vds at $130 \mathrm{~V}$ (a value within the failure range for $\mathrm{Kr}$ ), and incrementing Vgs by -1 to -2 volts. SEGR occurred in both samples between $-16 \mathrm{Vgs}$ and $-17 \mathrm{Vgs}$ or -18 Vgs. These data further support this apparent ion species effect, although the contribution of charge ionized within the highly-doped substrate cannot be ruled out at this point.

We tested a second pairing of ions (discussed in the full paper, but shown in Fig. 4). The data show a difference between the two ion species but this shift in the mean is not significant at the 95\% CL.

Lastly, we compare the bias necessary for SEGR under irradiation with $1405 \mathrm{MeV}$ Ag versus 2950 $\mathrm{MeV} \mathrm{Xe}$. Irradiations were performed at $-10 \mathrm{Vgs}$ bias on 3 samples per beam condition following the same procedures as before. Despite the Ag ions having a higher average LET throughout the epilayer and into a substantial portion of the drain substrate region (see Fig. 2), a higher applied Vds was necessary for SEGR to occur at $-10 \mathrm{Vgs}$ with Ag as compared with the heavier species, Xe (Fig. 5). This difference in failure threshold is significant at the $95 \% \mathrm{CL}$. This difference was further substantiated by irradiating 2 additional samples with $1405 \mathrm{MeV} \mathrm{Ag}$ at a drain bias of $50 \mathrm{Vds}$, near the mean of the threshold for SEGR from Xe. Both samples experienced SEGR at $-14 \mathrm{Vgs}$, having last survived at either $-12 \mathrm{~V}$ or $-13 \mathrm{~V}$.

To better understand these data, we also validated the Titus-Wheatley formula [3], $\mathrm{V}_{\mathrm{gs}}$ crit $=\left(10^{7}\right)\left(\mathrm{t}_{\mathrm{ox}}\right) /(1+\mathrm{Z} / 44)$, finding the critical Vgs for SEGR with Vds fixed at $0 \mathrm{~V}$ (Fig. 6). (Discussed in the paper, including fitting methods used; in the formula, $t_{o x}$ is the oxide thickness in $\mathrm{cm}, \mathrm{Z}$ is the ion atomic number.)

\section{DISCUSSION}

Past studies have suggested that the ion atomic number may play a role in SEGR susceptibility, beyond simply the ion LET or total charge ionization $[3,8]$. To our knowledge, this study is the first to control for the charge ionization in the silicon epilayer in order to examine the impact on SEGR of different ion species. Our results suggest that ion atomic number cannot be neglected when considering SEGR risk avoidance on orbit. 
The results presented in Figs. 3-5 have large error bars that represent the 95\% worst-case upper bound on the standard deviation for the distribution of failures. The small sample size, Vds interval, part-to-part variability, and the Poisson nature of the failure rate all contribute to this uncertainty in the best-fit standard deviation. Of these factors, the small sample size is likely the largest contributor, such that the significance of the results in this study would likely increase with more data. The impact of the other factors was lessened by a Vds increment of only $2.5 \%$ of the rated BVdss, a single lot-date-code for the samples, and a high fluence at each beam run.

As shown in Fig. 4, the higher mean threshold Vds for SEGR for $740 \mathrm{MeV} \mathrm{Ag} \mathrm{versus} 1618 \mathrm{MeV} \mathrm{Xe}$ was not significant at the $95 \%$ CL. In contrast, the $2950 \mathrm{MeV}$ Xe irradiations resulted in SEGR at a significantly lower Vds than did irradiations with lighter $1405 \mathrm{MeV} \mathrm{Ag}$ ions, despite the $\mathrm{Ag}$ ions depositing more energy in both the epilayer and the initial $70 \mu \mathrm{m}$ of highly-doped substrate (Figs. 2, 5). This result suggests that the lack of significance at the 95\% CL between $740 \mathrm{MeV} \mathrm{Ag}$ and $1618 \mathrm{MeV} \mathrm{Xe}$ may be due to the small sample size and the Vds interval size; alternatively, the effect of the ion species on the oxide may lessen as the average LET in the epilayer increases. Additional studies are needed to determine whether or when the energy deposition in the silicon dominates the species effects on the oxide or the importance of ion track structure within the epilayer.

We note that the variability in the Vds for SEGR was largest for the lightest ion tested. This variability may be due to a decreasing cross-section for SEGR as ions become lighter and/or deposit less energy. In addition, the $422 \mathrm{MeV} \mathrm{Cu}$ ions were obtained using a 2.8 mil Al degrader resulting in greater energy straggling. In the paper, we discuss and quantify these potential factors.

Charge ionized within the highly-doped drain substrate did not have as much of an effect as the charge in the epilayer or the ion atomic number on the SEGR failure threshold bias. In the case where Ag ionized more charge in both the epilayer and the first $70 \mu \mathrm{m}$ of the substrate than Xe, the heavier Xe ions ruptured the gate oxide at a lower drain-source bias. Only charge in the initial few $\mu \mathrm{m}$ of the heavilydoped substrate would be expected to contribute to the transient electric field due to the deformation of the epilayer/substrate interface drift field into the first few $\mu \mathrm{m}$ of the substrate at the location of the ion track due to the large concentration of charge ionized; however, in the majority of the substrate there is only a minimal electric field such that charge would be collected primarily by slower diffusion processes. Additionally, this charge would undergo comparatively higher recombination upon initial ionization. The important ion beam characteristics for inducing SEGR therefore are the total energy it can deposit in the epilayer including the epi/substrate interface region, and the ion atomic number.

Finally, we examine the additional Vgs bias required to reduce the threshold Vds of the lighter ion to the Vds threshold found for the heavier ion. In Fig. 3, a 6-V to 7-V increase in Vgs magnitude was required for $\mathrm{Cu}$ ions to induce SEGR at the $130 \mathrm{Vds}$ failure threshold determined for Kr. In Fig. 5, a 3-V to 4-V increase in Vgs magnitude was required for Ag ions to induce SEGR at the $50 \mathrm{Vds}$ threshold for Xe. From Fig. 6, we determine that at $0 \mathrm{Vds}$, the difference in Vgs magnitude for SEGR with $\mathrm{Cu}$ versus $\mathrm{Kr}$ is $4.6 \mathrm{~V}$, and with $\mathrm{Ag}$ versus $\mathrm{Xe}, 3 \mathrm{~V}$. This difference will be analyzed in the full paper in the context of the oxide response to the ion strike as well as ion track structure effects in the epilayer.

The work presented has SEGR hardness assurance implications which are also addressed in the paper.

\section{REFERENCES}

[1] T. A. Fischer, "Heavy-Ion-Induced, Gate-Rupture in Power MOSFETs," IEEE TNS, vol. 34, pp. 1786-1791, 1987.

[2] L. Scheick and L. Selva, "Sensitivity to LET and Test Conditions for SEE Testing of Power MOSFETs," in 2009 IEEE Radiation Effects Data Workshop, 2009, pp. 82-93.

[3] J. L. Titus, C. F. Wheatley, K. M. Van Tyne, J. F. Krieg, D. I. Burton, and A. B. Campbell, "Effect of ion energy upon dielectric breakdown of the capacitor response in vertical power MOSFETs," IEEE TNS, vol. 45, pp. 2492-2499, 1998.

[4] G. Busatto, F. Iannuzzo, A. Porzio, A. Sanseverino, F. Velardi, and G. Currò, "Experimental study of power MOSFET"s gate damage in radiation environment," Microelectronics and Reliability, vol. 46, pp. 1854-1857, 2006/11// 2006.

[5] D. Peyre, C. Poivey, C. Binois, R. Mangeret, G. Salvaterra, M. Beaumel, F. Pontoni, T. Bouchet, L. Pater, F. Bezerra, R. Ecoffet, E. Lorfevre, F. Sturesson, G. Berger, J. C. Foy, and B. Piquet, "SEGR Study on Power MOSFETs: Multiple Impacts Assumption," IEEE TNS, vol. 55, pp. 2181-2187, 2008. 
[6] P. F. Peyrard, T. Beutier, O. Serres, C. Chatry, R. Ecoffet, G. Rolland, D. Boscher, S. Bourdarie, C. Inguimbert, P. Calvel, and R. Mangeret, "A toolkit for space environment," in Radiation and Its Effects on Components and Systems, 2003. RADECS 2003. Proceedings of the 7th European Conference on, 2003, pp. 639-641.

[7] J. F. Ziegler and J. P. Biersack, "The Stopping and Range of Ions in Matter," http://www.srim.org.

[8] S. Liu, "Worst-Case Test Conditions of SEGR for Power DMOSFETs," IEEE TNS, vol. 57, pp. 279-287, 2010.

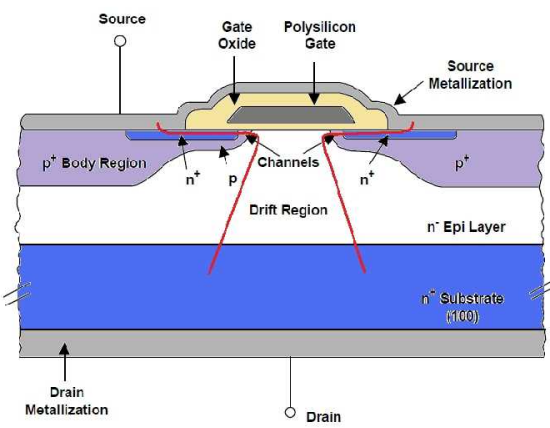

Figure 1. Illustration of a n-type VDMOS.

Table I. Ion Beam Properties for the 200V nVDMOS.

\begin{tabular}{|c|c|c|c|c|c|}
\hline Ion & Energy & Incident LET & LET at Oxide & $\begin{array}{c}\text { Mean LET in } \\
\text { Epi }\end{array}$ & $\begin{array}{c}\text { Total Charge } \\
\text { lonized in Epi }\end{array}$ \\
\hline$Z$ & $M e V$ & $M e V \cdot \mathrm{cm}^{2} / \mathrm{mg}$ & $\mathrm{MeV} \cdot \mathrm{cm}^{2} / \mathrm{mg}$ & $\mathrm{MeV} \cdot \mathrm{cm}^{2} / \mathrm{mg}$ & $p C$ \\
\hline 29 & 422 & 25.9 & 26.7 & 29.3 & 7.9 \\
\hline 36 & 1089 & 27.7 & 28.1 & 29.3 & 7.9 \\
\hline 47 & 740 & 53.8 & 55.5 & 57.8 & 15.5 \\
\hline 54 & 1618 & 54.6 & 55.4 & 57.8 & 15.5 \\
\hline 47 & 1405 & 42.7 & 43.4 & 45.4 & 12.2 \\
\hline 54 & 2950 & 41.5 & 41.8 & 42.8 & 11.5 \\
\hline
\end{tabular}



Figure 2. Ion LET as a function of penetration depth. Vertical dashed lines demarcate the epilayer region.

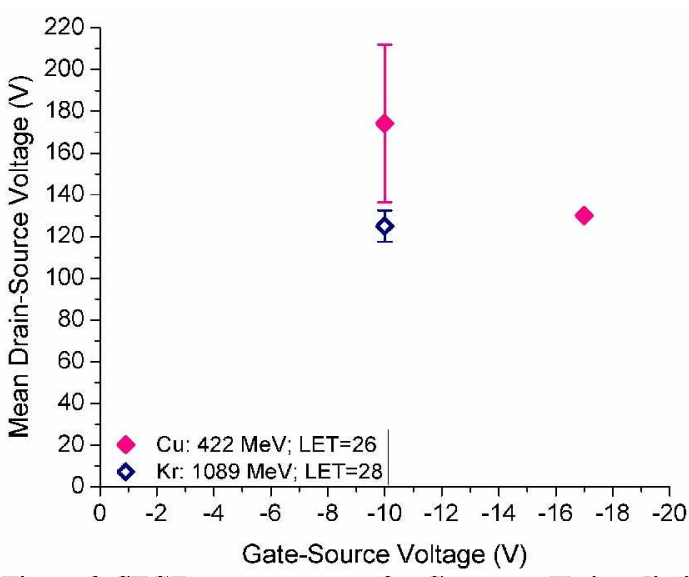

Figure 3. SEGR response curve for Cu versus $\mathrm{Kr}$ irradiation.



Figure 4. SEGR response curve for $A g$ versus $X e$, at incident LETs of $54 \mathrm{MeV} \cdot \mathrm{cm} 2 / \mathrm{mg}$.



Figure 5. SEGR response curves for $1405 \mathrm{MeV}$ Ag versus 2950 MeV Xe ions.

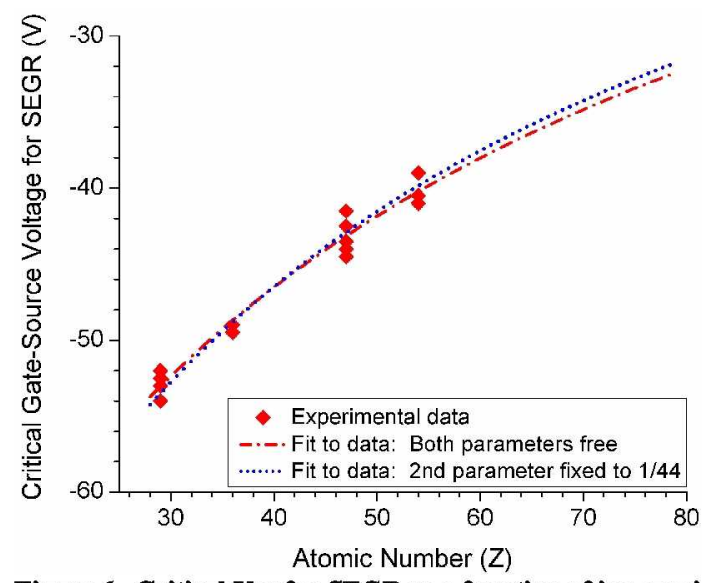

Figure 6. Critical Vgs for SEGR as a function of ion species. Data are fitted to the two-parameter reciprocal function $y=A /(1+B x)$, with either both parameters free, or with $B$ fixed to that of the Titus-Wheatley formula. 